\title{
Disc assessment and visual field analysis in a hospital glaucoma population
}

\author{
MARILYN BECK' AND ALEC G KARSERAS ${ }^{2}$ \\ From the 'University Hospital of Wales, Cardiff, and ${ }^{2}$ St Woolos Hospital, Newport
}

SUMMARY A hospital series of 432 patients with glaucoma was studied to quantify the ease of assessment of the cup/disc ratio and the visual fields. $19.9 \%$ of the discs were not easily visible to monitor accurately ophthalmoscopically and a further $27.9 \%$ were grossly cupped. $63 \%$ of the patients studied had general features or ocular pathology which might contribute to unreliable analysis of the visual field.

The 3 prime variables monitored during the management of glaucoma are the level of intraocular pressure, the appearance of the optic nerve head, and the state of the visual fields. It is usually the level of the intraocular pressure relative to the other 2 variables which determines the cardinal management decisions of either the initiation of life-long medical treatment or potentially sight-threatening drainage surgery.

In 'low-tension glaucoma' the visual field may deteriorate with intraocular pressures of less than $20 \mathrm{mmHg}$. An attempt to reduce this by drainage surgery may result in macular oedema and a dramatic fall in visual acuity. Thus a deteriorating visual field alone might not persuade all clinicians to risk macular function by surgery when the intraocular pressure is in the low-normal range. Hence the only absolute criterion for treatment or surgery in an eye with useful function is a very high intraocular pressure. In its absence decisions on management are a matter of individual clinical judgment, taking account of relative changes in these 3 main factors. Other features which influence the clinician include a family history of glaucoma, the age and general health of a patient and his life expectancy, pathological myopia, a central venous occlusion in the fellow eye, the total visual reserves, and the differences between the presenting and current intraocular pressures.

A decision on management is therefore based on the relative changes in the 3 prime variables with numerous background factors. The availability of accurate prime data when making the 2 cardinal management decisions can be of crucial importance.

This paper attempts to quantify within a short time scale in the life history of a hospital glaucoma popula-

Correspondence to Mr A. G. Karseras, FRCS. St Woolos Hospital, Newport, Gwent NPT 4SZ. tion 2 of these variables, namely, the availability and reliability of the optic disc appearance and the accuracy of visual field analysis.

\section{Materials and methods}

The ocular and general clinical status of 432 patients who are currently under observation for glaucoma was analysed from their hospital records. The data referring to the discs related to their clinic attendances in 1978. They were unselected and formed the bulk of the hospital glaucoma population.

There were 792 affected eyes (56 fellow eyes had normal discs and intraocular pressures, and 16 eyes had been enucleated). 496 eyes had chronic simple glaucoma, 77 had subacute angle closure. The remaining 219 were a mixed group consisting largely of patients with chronic closed-angle glaucoma, ocular hypertension (pressure $>30 \mathrm{mmHg}$ ), postuveitic glaucoma, and aphakic and post-traumatic glaucoma. The number of optic discs that could be assessed accurately with the ophthalmoscope (after attempted pupillary dilatation where necessary) was recorded. The number of eyes in which changes in the optic disc could contribute to management was quantified. Associated ocular and general clinical features adversely influencing the accuracy of visual field analysis were assessed. These patients had been tested with the Bjerrum screen or the Friedmann visual field analyser for central field defects. Peripheral fields were tested with the Aimark perimeter in most cases, and a small group was tested with the Goldmann perimeter. A substantial minority with gross field loss to confrontation had not been further tested. No attempt was made to relate any inaccuracy of analysis to the method of field testing. The inves- 
tigative effort was confined to the identification of associated ocular or general features militating against the reliability of future changes.

\section{Results}

OPTIC NERVE HEAD APPEARANCE

Availability of accurate data. In 158 of the 792 eyes with glaucoma examined it was not possible ophthalmoscopically to measure the cup/disc ratio accurately. The factors responsible for this were cataract (118 eyes), corneal opacity (5 eyes), central venous thrombosis (4 eyes). Extreme miosis with synechiae, high myopia, and congenitally abnormal discs prevented valid assessment in 31 eyes.

Relevance for future management. 221 eyes had grossly cupped discs with cup disc ratios of 0.8 or greater (maximum diameter of cup was taken irrespective of meridian). These discs were so cupped that their potential for further change was profoundly reduced.

Of the remaining 413 eyes 63 had gross field loss consisting of 2 or more quadrants lost to confrontation. In these eyes visual reserves were extremely low, and one would not normally wait for further deterioration in fields or disc appearance. Management was therefore based almost entirely on the level of intraocular pressure. A further 109 eyes had control of pressures at $18 \mathrm{mmHg}$ or less.

VISUAL FIELDS

This study was limited to the 573 eyes with chronic simple and subacute angle closure glaucoma. 361 eyes of the 573 examined had general and/or ocular features which made field analysis potentially unreliable.

(A) Associated ocular features adversely influencing the accuracy of visual field analysis. In 292 of these 573 eyes examined it was not possible to rely on field tests for evidence of progression of visual field loss to make decisions in management because of associated ocular pathology. The dominant factors responsible were: (1) lenticular: cataract and aphakia (215 eyes); the visual acuity was less than $6 / 12$ in the phakic eyes in this group, and a small minority were aphakic at the time of analysis; (2) maculopathy (65 eyes); (3) retinal vein occlusion (28 eyes); (4) other pathology (keratopathy, retinal detachment, pathological myopia, 5 eyes). Some of the eyes in 1-4 had more than the one condition.

(B) Associated general features adversely influencing the accuracy of visual field analysis. In 162 eyes of the 573 examined there were general features present which were a potential source of error in the use of field tests for evidence of progression of visual field loss. The chief factors responsible for this were: (1) senility (65 patients, 130 eyes); (2) extreme deafness
(10 patients, 20 eyes); (3) mental subnormality or illness (6 patients, 12 eyes).

Groups (A) and (B) were not mutually exclusive.

\section{Discussion}

The ideal patient with glaucoma is a reliable witness, with good concentration, is emmetropic with clear media, and has a healthy fundus and an easily described disc. In clinical practice such patients are a minority. This may not be so evident in a group of glaucoma patients studied only at presentation. The effects of increasing age and duration of glaucoma and its treatment result in a changing associated morbidity precluding the ideal situation. A hospital glaucoma population consists largely of such patients, and this study attempts to emphasise the limitations of their assessment.

Estimation of a reliable intraocular pressure was not found to present a significant problem. The occasional patient with bullous keratopathy or corneal scarring necessitated a Schi $\phi t z$ measurement rather than applanation tonometry. This was not so, however, with the other objectively obtained prime variable-the configuration of the optic nerve head. In 158 eyes $(19.9 \%)$ of the 792 glaucomatous eyes examined it was not possible to measure the cup/disc ratio. Ophthalmoscopically most of the 11 parameters of the optic disc given importance by Drance ${ }^{1}$ and the detailed changes of Hitchings and Spaeth ${ }^{2}$ would have been unavailable in a higher percentage of eyes. Likewise photography, stereophotography, stereogrammetry, and coloriometry would have been inapplicable to this group. Mydriatic examination with a fundus contact lens might have helped in assessing some of the discs in cataractous eyes in the earlier stages.

$221(27.9 \%)$ further eyes had grossly cupped discs with cup disc ratios of 0.8 or greater on direct ophthalmoscopy. As these discs were so cupped, it would be difficult to monitor the course of the glaucoma on the disc appearance, though serial photography if practical might have provided further information in selected cases. More reliance had to be placed either on field changes or on the level of intraocular pressure. It could be argued that discs with cups which extend to the edge on one side with a rim of one-fifth of a disc diameter on the other side could be monitored, but the wisdom of operating on such marginal disc changes alone is debatable.

Of the remaining 413 eyes 63 had extensive visual field loss ( 2 or more quadrants absent to confrontation). Disc appearances in these patients did not contribute to their management, as any residual ocular hypertension in isolation induced a decision for drainage surgery in eyes with advanced disease. It is 
not possible to define a precise level of 'residual hypertension'. This level would vary with the pressure found at initial presentation, possible contralateral venous thrombosis, and other background clinical features in eyes with such advanced disease.

Thus in $442(55.8 \%)$ of the 792 eyes details of the optic nerve head were unavailable or of doubtful contribution in management.

Of the remaining 350 eyes there were 109 in which the intraocular pressures were under $18 \mathrm{mmHg}$. At this level of intraocular pressure it would be expected that very few eyes would show increase in disc cupping. In those few eyes that did develop progressive disc cupping the possible advantages gained by reducing the pressure further would have to be balanced against the complications of intraocular surgery, namely, cataract formation and possible macular or disc oedema due to hypotony. In such a situation many clinicians would consider a slow natural erosion of visual reserves preferable to a risk of abrupt deterioration from surgical complications. Although optic disc changes in this group might occasionally indicate the need for phasing, checking for overenthusiastic treatment of hypertension, anaemia, or viscosity syndromes, these patients were in reality monitored by their intraocular pressure in isolation. Their discs were of potential value in management only if pressure control was lost.

At the time of analysis therefore in $551(69.5 \%)$ of the 792 eyes the optic disc appearance was not of great relevance in the management of the glaucoma.

The visual field changes are a subjective measurement and were also found to be potentially unreliable in a greater number of patients. In $292(50.9 \%)$ of the 573 eyes with a chronic simple or subacute angleclosure glaucoma there were associated ocular features militating against the accuracy and hence reliability of field testing. The commonest cause was cataract. The number of cataracts invalidating serial field analysis was found to be greater than that precluding accurate disc appraisal. The coexistence of cataract with glaucoma influencing the accuracy of visual field has been discussed previously. Miller and Karseras $^{3}$ and Playfair and Watson ${ }^{4}$ have noted an apparent worsening of visual field, which has subsequently improved after cataract extraction. The present analysis attempts to quantify this potential source of error. Cataract and other associated ocular morbidity will increase with duration of attendance at glaucoma clinics, and the relevance of serial refined visual field analysis in most patients with glaucoma can be seriously questioned. The more sophisticated the method of analysis, the more difficult it is to interpret the significance of the changes found in this group.

In addition to this potential source of error is the subjective nature of the response in a largely elderly population. Reliable perimetry was doubtful in 162 eyes of 573 reviewed because of the patient's general status. It was found that a total of 361 eyes of the 573 reviewed (63.0\%) had general and/or ocular features potentially contributing to unreliable field analysis.

The clinician should thus be aware that the data complex forming the basis of his decisions changes during the course of the disease, and may be or become greatly deficient in accuracy and availability because of the increasing associated morbidity and age changes.

We thank Robert Newcombe, PhD, who did the computer analyses.

\section{References}

1 Drance SM. Correlation of optic nerve head and visual field defects in glaucoma. Trans Ophthalmol Soc UK 1975; 95: 288-96.

2 Hitchings PA. Spaeth GL. The optic disc in glaucoma. I. Classification. Br J Ophthalmol 1976; 60: 778-85.

3 Miller SJH, Karseras AG. Blind registration and glaucoma simplex. Br J Ophthalmol 1974; 58: 455-61.

4 Playfair TJ, Watson PG. Management of chronic or intermittent primary angle-closure glaucoma: a long-term follow-up of the results of peripheral iridectomy used as an initial procedure. $\mathrm{Br} \mathrm{J}$ Ophthalmol 1979; 63: 23-8. 